\title{
Fibroblasts shape PDAC architecture
}

Pancreatic ductal adenocarcinomas (PDACs) are comprised of a desmoplastic stroma that surrounds tumour glandular structures (tumour glands). The bulk of this stroma is comprised of cancer-associated fibroblasts (CAFs), but the function of these CAFs in tumour progression is unclear, as both pro- and antitumorigenic functions have been reported. Primary PDAC cells are also known to have heterogeneous phenotypes, which might be driven by crosstalk with CAFs. To better understand the interplay between these cell types and the implications for PDAC progression, Ligorio, Sil et al. used a combination of singlecell RNA (scRNA) and proteomic techniques in both model systems and human PDAC tumours.

The authors began by analysing a PDAC cell line and a CAF cell line, which were fluorescently labelled so they could be distinguished, in co-culture experiments. The two cell types were co-cultured in different ratios to reflect clinical observations in PDAC. scRNA sequencing (scRNAseq) of PDAC cells cultured in a 10:90 ratio with CAFs showed that the PDAC cells highly expressed genes controlling proliferation (PRO) and epithelial-mesenchymal transition (EMT) compared with PDAC cell monocultures. This was consistent with the PRO and EMT phenotypes the authors had observed previously in mouse primary PDAC cells and circulating tumour cells. There was heterogeneous acquisition of these $\mathrm{PRO}$ and EMT programmes across the ratios of PDAC cells:CAFs, and some PDAC cells cultured at 10:90 expressed both PRO and EMT programmes (referred to as double-positive (DP) cells). scRNA-seq of CAFs indicated that they also had an increase in the PRO phenotype when cultured with PDAC cells, as well as upregulation of an inflammatory interferon response programme. Together, these data indicated that PDAC-CAF crosstalk drives the phenotype of both cell types.

To determine the molecular basis of this crosstalk, the authors treated several PDAC cell lines with conditioned media derived from CAFs. Protein levels of EMT (fibronectin 1 (FN1)) and PRO (Ki67) markers in the PDAC cells were consistent with the PRO, EMT and DP phenotypes observed at the RNA level. These markers reflected changes in cell functions: the majority of PDAC cell lines co-cultured with CAFs had higher proliferation and invasion rates in vitro. Furthermore, orthotopic implantation of a PDAC cell line and CAFs (10:90 ratio) together into immunodeficient mice significantly increased primary tumour growth and metastatic tumour burden compared with PDAC cells alone.

Further molecular analysis of the signalling pathways activated in PDAC cells indicated that both MEK-ERK and STAT3 pathways were activated in cultured cells and human primary tumours. Several lines of evidence suggested these pathways were functionally important, and pharmacological blockade of both in cultured cells suppressed proliferation and invasion. In addition, the authors identified seven factors secreted by CAFs that strongly correlated with DP programme induction in PDAC cells. One of these, transforming growth factor $\beta 1$ (TGF $\beta 1$ ), was crucial for PDAC cell proliferation and EMT.

To determine the relevance of the PRO and EMT phenotypes in human PDAC, RNA in situ hybridization (RNA-ISH) for FN1 and MKI67 (which encodes Ki67) was performed on core biopsy samples from 195 primary PDACs in a tissue microarray format. Using this new methodology, the authors analysed a total of 365 cores,

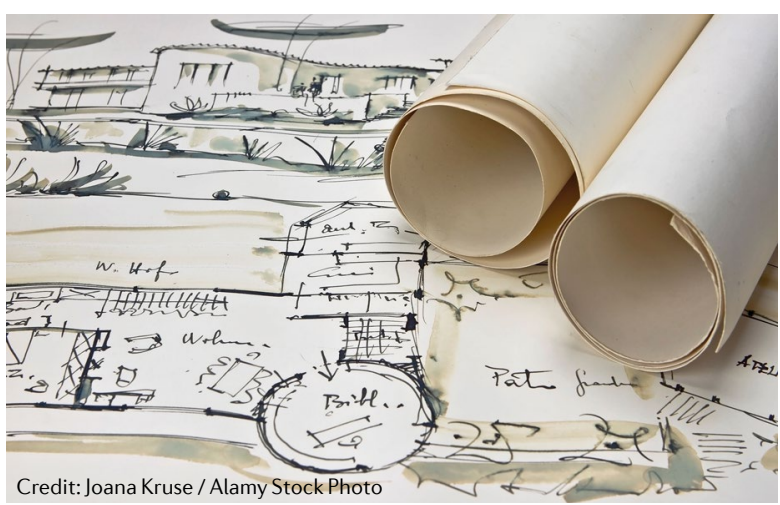

which encompassed 3,593 tumour glands; over 300,000 single cancer cells were analysed. Although most cells did not express either marker, $11.9 \%$ expressed MKI67 (PRO), $17.5 \%$ expressed FN1 (EMT) and $11.0 \%$ expressed both (DP). Normalization of each cell type overall to the total number of cells per patient did not provide much prognostic information, only that more PRO cells correlated with better survival. However, if spatial information was incorporated in the analysis through normalizing cell numbers per tumour gland, then a high number of DP cells correlated with worse survival, suggesting that important information is contained in the spatial architecture of PDACs.

To look further at this architecture, the authors first classified tumour glands according to the prevalence of the different cell types (PRO, EMT and DP), and observed substantial inter- and intrapatient heterogeneity. Furthermore, glands containing predominantly DP cells were enriched in tumours with high stromal content (>85\%), and those containing predominantly PRO cells were enriched in tumours of low stromal content $(<75 \%)$. In agreement with their previous survival analysis, a high prevalence of tumour glands containing predominantly DP cells, EMT cells or DP and EMT cells together were associated with poor survival. Finally, an analysis of patients who had been treated with chemotherapy indicated that those patients' tumours were enriched for glands containing predominantly EMT cells or DP and EMT cells together, suggesting that chemotherapy selects for, or cannot eradicate, these specific gland types.

Overall, this study highlights the importance of stromal context for understanding tumour cell heterogeneity, and the role of these interactions in shaping tumour architecture and patient prognosis.

Sarah Seton-Rogers

ORIGINAL ARTICLE Ligorio, M. \& Sil, S. et al. Stromal microenvironment shapes the intratumoral architecture of pancreatic cancer. Cell https://doi. org/10.1016/j.cell.2019.05.012 (2019) 